\title{
ON THE $r$-NUCLEARITY OF SOME INTEGRAL OPERATORS ON LEBESGUE SPACES
}

\author{
JULIO DELGADO \\ (Received September 20, 2013, revised February 14, 2014)
}

\begin{abstract}
In this paper we will exhibit a class of kernels generating $r$-nuclear operators. The class includes the Fox-Li and related operators. Estimates for the corresponding asymptotic behaviour of the eigenvalues are also derived.
\end{abstract}

1. Introduction. In this work we address to the problem of the membership to the ideal of $r$-nuclear operators for integral operators of the form

$$
T f(x)=\int_{-\alpha}^{\alpha} G(x) F(x y) H(y) f(y) d y,
$$

where $G$ and $H$ are bounded functions and $F$ is a smooth function admitting a suitable Taylor expansion. A such kind of operator arises in the theory of laser, particularly in the form of the Fox-Li operator which has attracted the interest of researchers in recent years (cf. [BBIN10], [BGI12], [BGHI12]). In those works, the authors were focused in the investigation of the asymptotic distribution of the singular values and eigenvalues. More recently, the $r$-nuclearity and its connection with Grothendieck-Lidskii's formula on compact Lie groups and applications to some differential operators have been studied in [DR13]. Applications of nuclear operators to the study of periodic pseudo-differential operators have been established in [DW13]. The nuclearity of operators on spaces of Bochner integrable functions (cf. [DE09]) and the corresponding relation with the martingale vector-maximal function for the study of traces have been considered in [Del]. Recent progress on the nuclearity and singular values can also be found in [FMO09] and [MO12].

The Fox-Li operator was introduced in [FL61], A. G. Fox and T. Li considered the repeated reflection of an electromagnetic wave between two plane-parallel rectangular mirrors. The current density at the surface of each reflector satisfies the eigenvalue integral equation

$$
T f(x)=\lambda f(x) .
$$

For a good account on the physics of such kind of operators we recommend the chapter XI, section 60 in the book [NE05] by Trefethen and Embree. The Fox-Li operator is defined by

$$
\left(\mathcal{F}_{\omega} f\right)(x):=\int_{-1}^{1} e^{i \omega(x-y)^{2}} f(y) d y, x \in(-1,1),
$$

2010 Mathematics Subject Classification. Primary 47B35; Secondary 47C05, 47B06, 45C05.

Key words and phrases. $r$-nuclear operators, Schatten-von Neumann ideals, trace formula, distribution of eigenvalues, Fox-Li operator. 
where $\omega$ is a positive real number. Since the kernel $k(x, y)=e^{i \omega(x-y)^{2}}$ is bounded this operator is compact, the kernel is $\operatorname{symmetric}(k(x, y)=k(y, x))$ but is not hard to see that the corresponding operator is not normal on $L^{2}([-1,1])$. The operators $(1.1)$ being compact they admit at most a countable set of eigenvalues with the origin as the only possible cluster point.

In [NM64] D. J. Newman and S. P. Morgan study the existence of eigenvalues for integral operators (1.1) when $F$ is an entire function of finite order with the aim of applying to the Fox-Li operator. Further studies on the problem of existence of eigenvalues for this kind of operators can be found in [NM64], [Coc65], [Hoc66], [SP61] and [CH74]. Numerical computations have recently appeared in [BBIN10] and [BIN11]. We do not consider the existence of eigenvalues in this work, we aim to the problem of finding sufficient conditions for the membership to the ideal of $r$-nuclear operators for operators of the form (1.1) on $L^{p}([-\alpha, \alpha])$. As a consequence some estimates for the distribution of eigenvalues are deducted.

The main concept we will employ in this work is that of $p$-nuclear operator on a Banach space. In this work we will refer to those operators as $r$-nuclear in order to distinguish with respect to the index of $L^{p}$ spaces. We recall that if $T$ is a compact operator on a complex Hilbert space $H$, the singular values of $T$ are the eigenvalues of the compact positive operator $|T|=\sqrt{T^{*} T}$ and will be denoted by $s_{n}(T)$. $T$ is called a trace class operator if the singular values are summable. If the singular values are square-summable $T$ is called a Hilbert-Schmidt operator. More generally, if $0<r<\infty$ and the sequence $\left(s_{n}(T)\right)_{n}$ is $r$ summable we say that $T$ belongs to the Schatten-von Neumann ideal $S_{r}(H)$ and it is well known that each $S_{r}(H)$ is an ideal in $\mathcal{L}(H)$.

The connection between the distribution of eigenvalues and the membership to an ideal of operators can be explained from the following inequality relating the eigenvalues and the singular values established by H. Weyl(cf. [Wey49]):

$$
\sum_{n=1}^{\infty}\left|\lambda_{n}(K)\right|^{r} \leq \sum_{n=1}^{\infty} s_{n}(K)^{r}, \quad r>0,
$$

where $K$ is a compact operator on a complex Hilbert space and $s_{n}(K)$ denotes the singular values of the operator $K$. The particular case $r=2$ goes back to I. Schur (1909)(cf. [Sch09]). We shall apply the notion of $p$-nuclear operators to deduce asymptotic behaviour of eigenvalues for our operators (1.1). For a recent account on the state of the art of $p$-nuclear operators see [HP10].

2. $r$-nuclearity and the asymptotic behaviour of eigenvalues. In this section we begin by recalling some basic facts about the concepts of nuclear, $r$-nuclearity and the trace on Banach spaces. In particular we shall be interested in $L^{p}(\mu)$ spaces. We refer the reader to [Pie80] and the Chapter 4.2 of [Pie87] for the general theory of traces on operator ideals and the basic notation used in this section, see also [GGK00] for an account on the asymptotic behaviour of eigenvalues. After those preliminaries we will state the results.

Let $E$ be a Banach space which has the approximation property (cf. [Pie87]). It is well known that the spaces $L^{p}(\Omega, \mathcal{M}, \mu)$ satisfy the approximation property for any measure $\mu$ 
and $1 \leq p \leq \infty$ (cf. [Pie80, Lemma 19.3.5]). Thus, a Banach space $E$ is said to have the approximation property if for every compact subset $K$ of $E$ and every $\varepsilon>0$ there exists a finite rank bounded operator $B$ on $E$ such that

$$
\|x-B x\|_{E}<\varepsilon \text { for all } x \in K .
$$

Let $0<r \leq 1$ and $T$ be an operator from $E$ into $E$, we will say that $T$ is $r$-nuclear if it admits a representation

$$
T=\sum_{n=1}^{\infty} \varphi_{n} \otimes f_{n}, \quad\left(\varphi_{n} \in E, f_{n} \in E^{\prime}\right)
$$

such that

$$
\sum_{n=1}^{\infty}\left\|\varphi_{n}\right\|_{E}^{r}\left\|f_{n}\right\|_{E^{\prime}}^{r}<\infty .
$$

The class of $r$-nuclear operators is endowed with the quasi-norm

$$
n_{r}(T)=\inf \left\{\sum_{n=1}^{\infty}\left\|\varphi_{n}\right\|_{E}^{r}\left\|f_{n}\right\|_{E^{\prime}}^{r}\right\}^{\frac{1}{r}},
$$

where the inf is taken over the representations of $T$ such that (2.1) holds. The important case $r=1$ corresponds to the Ruston-Grothendieck algebra of nuclear operators where one can define the concept of trace, in this case $n_{1}$ is a norm. Note also that every $r$-nuclear operator is also nuclear. It is well known that in the setting of Hilbert spaces those operators constitute the trace class operator ideal or the Schatten-von Neumann ideal $S_{1}$. A more general fact is that in the setting of Hilbert spaces the class of $r$-nuclear operators agrees with the Schattenvon Neumann ideal of order $r$, a result due to R. Oloff (cf. [Olo72]). In [Pie84] A. Pietsch proved that for $r>1$ the concept of $r$-nuclear operator loses sense.

The set of nuclear operators from $E$ into $E$ forms the ideal of nuclear operators $\mathfrak{N}(E)$ endowed with the norm $n_{1}$. In order to ensure the existence of a good definition of the trace on the ideal of nuclear operators $\mathfrak{N}(E)$ one is constraints to consider Banach spaces $E$ enjoying the approximation property. In such a case, if $T: E \rightarrow E$ is nuclear, the trace is defined by

$$
\operatorname{tr}(T)=\sum_{n=1}^{\infty} x_{n}^{\prime}\left(y_{n}\right)
$$

where $T=\sum_{n=1}^{\infty} x_{n}^{\prime} \otimes y_{n}$ is a representation of $T$, and it can be shown that this definition is independent of the representation(cf. [Pie87]).

The case $r=2 / 3$ is a special one, A. Grothendieck proved (cf. [Gro55]) that the trace of a 2/3-nuclear operator agrees with the sum of all the eigenvalues with multiplicities counted. This coincidence is known as Lidskii's formula (cf. [Lid59]) which holds on Hilbert spaces. The question about the sharpness of the index $r=2 / 3$ in the concrete case of $L^{p}$ spaces has been recently considered in [RL13]. The membership to the class of $r$-nuclear operators can be used to deduce properties on the asymptotic behaviour of the corresponding operators by 
virtue of the next theorem coming from the theory of operator ideals (cf. [Gro55] Chapter II, p. 16, [Pie87] Theorem 3.8.6, [GGK00] Chapter 5, Theorem 4.2):

THEOREM 1. Let $E$ be a Banach space which has the approximation property. Let $T$ be an $r$-nuclear operator from $E$ into $E$ for some $0<r \leq 1$. Then

$$
\sum_{n=1}^{\infty}\left|\lambda_{n}(T)\right|^{t} \leq n_{r}(T)^{t}, \quad \frac{1}{t}=\frac{1}{r}-\frac{1}{2}
$$

where $\lambda_{n}(T)$ denotes the eigenvalues of $T$ multiplicities counted.

Theorem 1 was established by Grothendieck [Gro55], and extended by e.g. König ([Kön78, page 107]) to the scale of Lorentz sequences spaces; see also [Pie87, Theorem 3.8.6]. We will applied the Theorem 1 joint with some sufficient conditions for $r$-nuclearity in order to obtain estimates on the distribution of eigenvalues. In the case of $L^{p}(\mu)$ spaces for $\sigma$-finite measures a characterisation of nuclear operators is avalaible in terms of a kernel representation, see [Del10a] and [Del10b]. The following theorem is an immediate consequence of Theorem 2.2 in [Del10a] and will be applied in the particular case of the Lebesgue measure on a finite interval in the real line.

THEOREM 2. Let $\left(\Omega_{i}, \mathcal{M}_{i}, \mu_{i}\right)(i=1,2)$ be two measure spaces with finite measures $\mu_{1}$ and $\mu_{2}$ respectively. Let $1 \leq p_{1}, p_{2}<\infty, 0<r \leq 1$ and let $q_{1}$ be such that $\frac{1}{p_{1}}+\frac{1}{q_{1}}=1$. An operator $T: L^{p_{1}}\left(\mu_{1}\right) \rightarrow L^{p_{2}}\left(\mu_{2}\right)$ is $r$-nuclear if and only if there exist sequences $\left(g_{n}\right)_{n}$ in $L^{p_{2}}\left(\mu_{2}\right)$, and $\left(h_{n}\right)_{n}$ in $L^{q_{1}}\left(\mu_{1}\right)$ such that $\sum_{n=1}^{\infty}\left\|g_{n}\right\|_{L^{p_{2}}}^{r}\left\|h_{n}\right\|_{L^{q_{1}}}^{r}<\infty$, and for all $f \in$ $L^{p_{1}}\left(\mu_{1}\right)$ :

$$
T f(x)=\int\left(\sum_{n=1}^{\infty} g_{n}(x) h_{n}(y)\right) f(y) d \mu_{1}(y), \text { for a.e } x .
$$

We are now ready to exhibit our class of $r$-nuclear operators which will be generated by suitable $C^{\infty}$ kernels. In this paper the term smooth will refer to $C^{\infty}$ functions. The main technique is to take advantage of corresponding MacLaurin series for the kernels.

THEOREM 2.1. Let $\alpha>0$ be a real number, $0<r \leq 1$ and $1 \leq p<\infty$. Let $F:(-\beta, \beta) \rightarrow \mathbb{C}$ be a function having a MacLaurin series satisfying $\left|D^{n} F(0)\right| \leq M n(n !)^{s}$, $n=0,1, \ldots$, for some real $M$ and $0<s<1$. If $G, H:(-\alpha, \alpha) \rightarrow \mathbb{C}$ are bounded functions with $\alpha^{2} \leq \beta$, then the kernel $(x, y) \in(-\alpha, \alpha) \times(-\alpha, \alpha) \rightarrow G(x) F(x y) H(y) \in \mathbb{C}$ begets an $r$-nuclear operator $T$ on $L^{p}([-\alpha, \alpha])$ such that the Lidskii's formula holds for $T$,

$$
n_{r}^{r}(T) \leq\left(2 \alpha M\|G\|_{L^{\infty}}\|H\|_{L^{\infty}}\right)^{r} \sum_{n=0}^{\infty} \frac{\alpha^{2 r n}}{n !^{(1-s) r}} \text { and } \operatorname{tr}(T)=\int_{-\alpha}^{\alpha} G(x) F\left(x^{2}\right) H(x) d x .
$$

ProOF. We can write

$$
F(t)=\sum_{j=0}^{\infty} \frac{D^{j} F(0)}{j !} t^{j}, \quad \text { for all } t \in(-\beta, \beta) .
$$


If $(x, y) \in(-\alpha, \alpha) \times(-\alpha, \alpha)$ we have

$$
F(x y)=\sum_{j=0}^{\infty} \frac{D^{j} F(0)}{j !}(x y)^{j}=\sum_{j=0}^{\infty} \frac{D^{j} F(0)}{j !} x^{j} y^{j} .
$$

The condition $\alpha^{2} \leq \beta$ implies that $F(x y)$ is well defined for $(x, y) \in(-\alpha, \alpha) \times(-\alpha, \alpha)$. We define

$$
g_{j}(x)=\frac{D^{j} F(0)}{(j !)^{s}} x^{j}, \quad h_{j}(y)=\frac{y^{j}}{(j !)^{\ell}},
$$

where $\ell=1-s$. We shall calculate $\left\|g_{j}\right\|_{L^{p}},\left\|h_{j}\right\|_{L^{q}}\left(\frac{1}{p}+\frac{1}{q}=1\right)$. We first consider $1<p<$ $\infty$ :

In a similar way we get

$$
\left\|g_{j}\right\|_{L^{p}}=2^{\frac{1}{p}} \frac{\left|D^{j} F(0)\right|}{(j !)^{S}} \frac{\left(\alpha^{(p j+1)}\right)^{\frac{1}{p}}}{(p j+1)^{\frac{1}{p}}} .
$$

Hence

$$
\left\|h_{j}\right\|_{L^{q}}=2^{\frac{1}{q}} \frac{\left(\alpha^{(q j+1)}\right)^{\frac{1}{q}}}{(j !)^{\ell}(q j+1)^{\frac{1}{q}}} .
$$

$$
\begin{aligned}
\left\|g_{j}\right\|_{L^{p}}\left\|h_{j}\right\|_{L^{q}} & =2 \frac{\left|D^{j} F(0)\right|}{(j !)^{s}} \frac{\left(\alpha^{(p j+1)}\right)^{\frac{1}{p}}}{(p j+1)^{\frac{1}{p}}} \frac{\left(\alpha^{(q j+1)}\right)^{\frac{1}{q}}}{(j !)^{\ell}(q j+1)^{\frac{1}{q}}} \\
& =2 \frac{\left|D^{j} F(0)\right|}{(j !)^{s}} \frac{\alpha^{(2 j+1)}}{(p j+1)^{\frac{1}{p}}(q j+1)^{\frac{1}{q}}(j !)^{\ell}} \leq 2 \alpha M \frac{\alpha^{2 j}}{j !^{\ell}} .
\end{aligned}
$$

The last inequality has been obtained from the hypothesis on the estimation of the derivatives $D^{j} F(0)$ and the fact that

$$
(p j+1)^{\frac{1}{p}}(q j+1)^{\frac{1}{q}} \geq p^{\frac{1}{p}} j^{\frac{1}{p}} q^{\frac{1}{p}} j^{\frac{1}{q}} \geq j .
$$

Now, since $G$ and $H$ are bounded we obtain

$$
\sum_{n=0}^{\infty}\left\|G g_{n}\right\|_{L^{p}}^{r}\left\|H h_{n}\right\|_{L^{q}}^{r} \leq\left(2 \alpha M\|G\|_{L^{\infty}}\right)^{r}\|H\|_{L^{\infty}}^{r} \sum_{n=0}^{\infty} \frac{\alpha^{2 r n}}{(n !)^{\ell r}}<\infty .
$$

From the characterisation of $r$-nuclear operators (Theorem 2), the kernel $k(x, y)=$ $G(x) F(x y) H(y)$ begets an $r$-nuclear operator from $L^{p}([-\alpha, \alpha])$ into $L^{p}([-\alpha, \alpha])$ and the estimation of the $r$-nuclear quasi-norm follows from the last inequality. If $p=1$, similar calculations to the case $p>1$ lead to the following

$$
\left\|g_{j}\right\|_{L^{1}}^{r}\left\|h_{j}\right\|_{L^{\infty}}^{r} \leq(2 \alpha M)^{r} \frac{\alpha^{2 r j}}{(j !)^{\ell r}} .
$$

The $r$-nuclearity follows from this inequality as well as the estimation of the $r$-nuclear quasinorm. The formula for the trace follows from integration on the diagonal and the RustonGrothendieck's definition. In fact, our kernel is well behaved along the diagonal and the 
integral in absolute value $\int_{-\alpha}^{\alpha}|k(x, x)| d x$ is controlled by the nuclear norm $(r=1)$ :

$$
n_{1}(T) \leq 2 \alpha M\|G\|_{L^{\infty}}\|H\|_{L^{\infty}} \sum_{n=0}^{\infty} \frac{\alpha^{2 n}}{(n !)^{1-s}}<\infty .
$$

The operator $T$ is $r$-nuclear for $r \leq 2 / 3$, hence the Lidskii's formula holds.

REMARK 2.2. Not every kernel is convenient to calculate the trace integrating along the diagonal due to the degeneracy of the measure on it. When the kernel is representable by an expansion of the kind appearing in Theorem 2.1 one is allowed to proceed in such a way. For a general kernel the integration along the diagonal should be calculated involving an averaging processes, see e.g. [Del10a], [Del10b].

EXAMPLE. The condition on the derivatives in Theorem 2.1 can be improved if $0<$ $\alpha<1$. One can replace the condition on the derivatives by $\left|D^{n} F(0)\right| \leq M(n+1)$ ! taking advantage of the convergence of the geometric series $\sum_{n=0}^{\infty} \alpha^{\tau n}$ for a suitable $\tau$ in the proof. This extension allow us to consider for example the function $F:[0, \alpha] \rightarrow \mathbb{R}$ given by $F(t)=\frac{1}{1-t}$ for $0<\alpha<1$. In this case $D^{n} F(0)=n$ !, since $F$ is given by the geometric series $\sum_{n=0}^{\infty} t^{n}$. One can write $k(x, y)=\sum_{n=0}^{\infty} x^{n} y^{n}$ and follow a similar argument as above.

If $0<\alpha<1$ and $\alpha^{2} \leq \beta$, we can remove the condition on the derivatives at 0 since they can be deduced from the convergence of the MacLaurin series. In fact, from the convergence of

$$
\sum_{n=0}^{\infty} \frac{D^{n} F(0)}{n !} t^{n}
$$

for $t=1$, we have $\lim _{n} \frac{\left|D^{n} F(0)\right|}{n !}=0$ and the boundedness of the sequence $\frac{\left|D^{n} F(0)\right|}{n !}$ follows. The convergence of the geometric series $\sum_{n=0}^{\infty} \alpha^{\tau n}$ for a suitable $\tau$ leads to the following corollary.

COROLlary 2.3. Let $0<\alpha<1$ be a real number, $0<r \leq 1$ and $1 \leq p<$ $\infty$. Let $F:(-\beta, \beta) \rightarrow \mathbb{C}$ be a function having a MacLaurin series with $\alpha^{2} \leq \beta$. If $G, H:(-\alpha, \alpha) \rightarrow \mathbb{C}$ are bounded functions, then the kernel $(x, y) \in(-\alpha, \alpha) \times(-\alpha, \alpha) \rightarrow$ $G(x) F(x y) H(y) \in \mathbb{C}$ begets an $r$-nuclear operator $T$ on $L^{p}([-\alpha, \alpha])$ such that the Lidskii's formula holds for $T$,

$$
n_{r}^{r}(T) \leq\left(2 \alpha M\|G\|_{L^{\infty}}\|H\|_{L^{\infty}}\right)^{r} \sum_{n=0}^{\infty} \frac{\alpha^{2 r n}}{n^{r}} \text { and } \operatorname{tr}(T)=\int_{-\alpha}^{\alpha} G(x) F\left(x^{2}\right) H(x) d x .
$$

We now formulate a theorem in terms of classical sufficient condition for the convergence of Taylor series. This kind of hypothesis allows us to improve the estimate of the quasi-norm with respect to Theorem 2.1 .

THEOREM 2.4. Let $\alpha>0$ be a real number, $0<r \leq 1$ and $1 \leq p<\infty$. Let $F:(-\beta, \beta) \rightarrow \mathbb{C}$ be a smooth function such that $\alpha^{2} \leq \beta,\left|D^{n} F(x)\right| \leq M A^{n}$ for every 
$n \in \mathbb{N}$ and $x \in I$. If $G, H:(-\alpha, \alpha) \rightarrow \mathbb{C}$ are bounded functions, then the kernel $(x, y) \in$ $(-\alpha, \alpha) \times(-\alpha, \alpha) \rightarrow G(x) F(x y) H(y) \in \mathbb{C}$ begets an $r$-nuclear operator $T$ on $L^{p}([-\alpha, \alpha])$ such that the Lidskii's formula holds for $T$,

$$
n_{r}^{r}(T) \leq\left(2 \alpha M\|G\|_{L^{\infty}}\|H\|_{L^{\infty}}\right)^{r} \sum_{n=0}^{\infty} \frac{\left(A \alpha^{2}\right)^{r n}}{(n(n !))^{r}}, \quad \operatorname{tr}(T)=\int_{-\alpha}^{\alpha} G(x) F\left(x^{2}\right) H(x) d x,
$$

and

$$
\sum_{n=1}^{\infty}\left|\lambda_{n}(T)\right|^{t}<\infty, \quad t \in(0,2] .
$$

Proof. The condition on the derivatives ensures the convergence of the Taylor series of $F$ to $F(x)$ in $I$. We define $g_{j}(x)=\frac{D^{j} F(0)}{j !} x^{j}, h_{j}(y)=y^{j}$, in the Taylor expansion in the proof of Theorem 2.1. Let $r$ be such that $0<r \leq 1$, we obtain

$$
\begin{aligned}
\left\|g_{j}\right\|_{L^{p}}^{r}\left\|h_{j}\right\|_{L^{q}}^{r} & =\left(2 \frac{\left|D^{j} F(0)\right|}{j !} \frac{\alpha^{(2 j+1)}}{(p j+1)^{\frac{1}{p}}(q j+1)^{\frac{1}{q}}}\right)^{r} \\
& \leq(2 \alpha M)^{r}\left(\frac{A^{j} \alpha^{2 j}}{j(j !)}\right)^{r} .
\end{aligned}
$$

Hence

$$
\sum_{n=0}^{\infty}\left\|G g_{j}\right\|_{L^{p}}^{r}\left\|H h_{j}\right\|_{L^{q}}^{r} \leq\left(2 \alpha M\|G\|_{L^{\infty}}\|H\|_{L^{\infty}}\right)^{r} \sum_{n=0}^{\infty} \frac{\left(A \alpha^{2}\right)^{r n}}{(n(n !))^{r}}<\infty .
$$

Note that from Theorem 1 the series

$$
\sum_{n=1}^{\infty}\left|\lambda_{n}(T)\right|^{t}
$$

converges for $\frac{1}{t}=\frac{1}{r}-\frac{1}{2}$. Hence for all $t$ in the range $(0,2]$. Since, the operator is in particular $r$-nuclear for $r \leq 2 / 3$ the Lidskii's formula follows from Grothendieck's theorem (cf. [Gro55]) for 2/3-nuclear operators. The trace formula follows as in Theorem 2.1.

REMARK 2.5. (i) We have seen that under the hypothesis of Theorem 2.4 we dispose of the following comparison for the eigenvalues of the operator $T$ on $L^{p}$ :

$$
\sum_{n=1}^{\infty}\left|\lambda_{n}(T)\right|^{\frac{2 r}{2-r}} \leq\left(2 \alpha M\|G\|_{L^{\infty}}\|H\|_{L^{\infty}}\right)^{\frac{2 r}{2-r}}\left(\sum_{n=0}^{\infty} \frac{\left(A \alpha^{2}\right)^{r n}}{(n(n !))^{r}}\right)^{\frac{2}{2-r}} .
$$

(ii) When $p=2$ the ideal of $r$-nuclear operator coincides with the Schatten-von Neumann ideal $S_{r}$. Hence, under the assumptions of Theorem 2.4 the corresponding operator $T$ belongs to $S_{r}\left(L^{2}([-\alpha, \alpha])\right)$ for all $0<r \leq 1$.

The function $F(t)=e^{-2 i \omega t}$ appearing in the kernel of the Fox-Li operator has bounded derivatives. The theorem above with $A=1$ implies the following corollary.

COROLlary 2.6. Let $\alpha>0$ be a real number, $0<r \leq 1$ and $1 \leq p<\infty$. Let $F:(-\beta, \beta) \rightarrow \mathbb{C}$ be a smooth function such that $\alpha^{2} \leq \beta,\left|D^{n} F(x)\right| \leq M$ for every 
$n \in \mathbb{N}$ and $x \in I$. If $G, H:(-\alpha, \alpha) \rightarrow \mathbb{C}$ are bounded functions, then the kernel $(x, y) \in$ $(-\alpha, \alpha) \times(-\alpha, \alpha) \rightarrow G(x) F(x y) H(y) \in \mathbb{C}$ begets an $r$-nuclear operator $T$ on $L^{p}([-\alpha, \alpha])$ such that the Lidskii's formula holds for $T$,

$$
n_{r}^{r}(T) \leq\left(2 \alpha M\|G\|_{L^{\infty}}\|H\|_{L^{\infty}}\right)^{r} \sum_{n=0}^{\infty} \frac{\alpha^{2 r n}}{(n(n !))^{r}}, \operatorname{tr}(T)=\int_{-\alpha}^{\alpha} G(x) F\left(x^{2}\right) H(x) d x,
$$

and

$$
\sum_{n=1}^{\infty}\left|\lambda_{n}(T)\right|^{t}<\infty, t \in(0,2] .
$$

An improvement of the corollary above for the estimation of the $r$-nuclear quasi-norm can be obtained when $F(x y)=e^{-2 i \omega x y}$ in (1.1).

COROllary 2.7. Let $\omega>0$ be a real number, $0<r \leq 1$ and $1 \leq p<\infty$. If $G, H:(-1,1) \rightarrow \mathbb{C}$ are bounded functions, then the kernel $(x, y) \in(-1,1) \times(-1,1) \rightarrow$ $G(x) e^{-2 i \omega x y} H(y) \in \mathbb{C}$ begets an $r$-nuclear operator $T_{\omega}$ on $L^{p}([-1,1])$ such that the Lidskii's formula holds for $T_{\omega}$,

$$
n_{r}^{r}\left(T_{\omega}\right) \leq\left(4 C\|G\|_{L^{\infty}}\|H\|_{L^{\infty}}\right)^{r} \sum_{n=0}^{\infty}\left(\frac{(2 \omega)^{2 n}}{(2 n) !}\right)^{r},
$$

where $C$ is the corresponding constant for the quasi-triangle inequality of the quasi-norm $n_{r}$.

PROOF. We start from the explicite Taylor series for the real part and imaginary part of $F(x y)=e^{-2 i \omega x y}$ :

$$
\begin{aligned}
& \sin (2 \omega x y)=\sum_{n=1}^{\infty}(-1)^{n-1} \frac{(2 \omega x y)^{2 n-1}}{(2 n-1) !}, \\
& \cos (2 \omega x y)=\sum_{n=0}^{\infty}(-1)^{n} \frac{(2 \omega x y)^{2 n}}{(2 n) !} .
\end{aligned}
$$

For the term $\sin (2 \omega x y)$ and considering the particular case $\alpha=1$ in the proof of Theorem 2.4 , we define $g_{n}(x)=\frac{(-1)^{n-1}}{(2 n-1) !}(2 \omega x)^{2 n-1}$ and $h_{n}(y)=y^{2 n-1}$. We shall only consider $p>1$, the corresponding estimations for $p=1$ are similar:

$$
\begin{aligned}
\left\|g_{n}\right\|_{L^{p}}^{r}\left\|h_{n}\right\|_{L^{q}}^{r} & =\left(2 \frac{(2 \omega)^{2 n-1}}{(2 n-1) !(p(2 n-1)+1)^{\frac{1}{p}}(q(2 n-1)+1)^{\frac{1}{q}}}\right)^{r} \\
& \leq 2^{r}\left(\frac{(2 \omega)^{2 n-1}}{(p(2 n-1)+1)^{\frac{1}{p}}(q(2 n-1)+1)^{\frac{1}{q}}(2 n-1) !}\right)^{r} .
\end{aligned}
$$

Since $p, q>1$ and $\frac{1}{p}+\frac{1}{q}=1$ the term into the parenthesis in the denominator satisfies

$$
(p(2 n-1)+1)^{\frac{1}{p}}(q(2 n-1)+1)^{\frac{1}{q}}(2 n-1) ! \geq(2 n) ! .
$$

Hence 


$$
\begin{aligned}
& \sum_{n=1}^{\infty}\left\|G g_{n}\right\|_{L^{p}}^{r}\left\|H h_{n}\right\|_{L^{q}}^{r} \leq\left(2\|G\|_{\left.L^{\infty}\|H\|_{L^{\infty}}\right)^{r}} \sum_{n=1}^{\infty}\left(\frac{(2 \omega)^{2 n-1}}{(2 n) !}\right)^{r}\right. \\
& <\infty \text {. }
\end{aligned}
$$

A similar estimate holds for the part of the kernel corresponding to $\cos (2 \omega x y)$, one has

$$
\begin{aligned}
& \sum_{n=1}^{\infty}\left\|G g_{n}\right\|_{L^{p}}^{r}\left\|H h_{n}\right\|_{L^{q}}^{r} \leq\left(2\|G\|_{L^{\infty}}\|H\|_{L^{\infty}}\right)^{r} \sum_{n=0}^{\infty}\left(\frac{(2 \omega)^{2 n}}{(2 n) !(2 n p+1)^{\frac{1}{p}}(2 n q+1)^{\frac{1}{q}}}\right)^{r} \\
& \leq\left(2\|G\|_{L^{\infty}}\|H\|_{L^{\infty}}\right)^{r} \sum_{n=0}^{\infty}\left(\frac{(2 \omega)^{2 n}}{(2 n) !}\right)^{r} \text {. }
\end{aligned}
$$

We now observe that $T_{\omega}=T_{\omega}^{(1)}+i T_{\omega}^{(2)}$, where $T_{\omega}^{(1)}, T_{\omega}^{(2)}$ are integral operators with respective kernels $\cos (2 \omega x y)$ and $\sin (2 \omega x y)$. From the corresponding inequalities for $r$-nuclear quasinorms of $T_{\omega}^{(1)}$ and $T_{\omega}^{(2)}$ we obtain

$$
n_{r}^{r}\left(T_{\omega}\right) \leq\left(4 C\|G\|_{L^{\infty}}\|H\|_{L^{\infty}}\right)^{r} \sum_{n=0}^{\infty}\left(\frac{(2 \omega)^{2 n}}{(2 n) !}\right)^{r}<\infty .
$$

The constant $C$ is the corresponding quasi-triangle inequality for the quasi-norm $n_{r}$. The $r$-nuclearity and the estimation of the quasi-norm follow from the last inequality.

Acknowledgements. The author was supported by a Marie Curie International Incoming Fellowship within the 7th European Community Framework Programme under grant PIIF-GA-2011-301599. The author also wants to thanks an anonymous referee for his valuable comments and corrections which improved the presentation of this manuscript.

\section{REFERENCES}

[BBIN10] A. Böttcher, H. Brunner, A. Iserles AND S. P. Norsett, On the singular values and eigenvalues of the Fox-Li and related operators, New York J. Math. 16 (2010), 539-561.

[BGHI12] A. Böttcher, S. Grudsky, D. Huybrechs And A. Iserles, First-order trace formula for the iterates of the Fox-Li operator, A panorama of modern operator theory and related topics, 207-224, Oper. Theory Adv. Appl. 218, Birkhäuser/Springer Basel AG, Basel, 2012.

[BGI12] A. Böttcher, S. Grudsky and A. Iserles, The Fox-Li operator as a test and a spur for Wiener-Hopf theory, Essays in mathematics and its applications, 37-48, Springer, Heidelberg, 2012.

[BIN11] H. BRUnNer, A. IsERLES AND S. NøRSETT, The computation of the spectra of highly oscillatory Fredholm integral operators, J. Integral Equations Appl. 23 (2011), 467-519.

[CH74] J. A. COCHRAN AND E. HINDS, Eigensystems associated with the complex-symmetric kernels of laser theory, SIAM J. Appl. Math. 26 (1974), 776-786.

[Coc65] J. A. Cochran, The existence of eigenvalues for the integral equations of laser theory, Bell System Tech. J. 44 (1965), 77-88.

[DE09] A. Deitmar ANd S. Echterhoff, Principles of harmonic analysis, Universitext, Springer, 2009.

[Del] J. DELGADo, Trace formulas for nuclear operators in spaces of Bochner integrable functions, Monatsh. Math. 172 (2013), 259-275. 
[Del10a] J. Delgado, A trace formula for nuclear operators on $L^{p}$, In Pseudo-differential operators: complex analysis and partial differential equations, 181-193, Oper. Theory Adv. Appl. 205, Birkhäuser Verlag, Basel, 2010.

[Del10b] J. Delgado, The trace of nuclear operators on $L^{p}(\mu)$ for $\sigma$-finite Borel measures on second countable spaces, Integral Equations Operator Theory 68 (2010), no. 1, 61-74.

[DR13] J. Delgado AND M. RUZHAnsky, Lp-nuclearity, traces, and Grothendieck-Lidskii formula on compact Lie groups, J. Math. Pures. Appl. (9) 102 (2014), no. 1, 153-172.

[DW13] J. Delgado And M. W. Wong, $L^{p}$-nuclear pseudo-differential operators on $\mathbb{Z}$ and $\mathbb{S}^{1}$, Proc. Amer. Math. Soc. 141 (2013), no. 4, 3935-3942.

[FL61] A. FoX AND T. LI, Resonant modes in a maser interferometer, Bell Syst. Tech. J. 40 (1961), $453-488$.

[FMO09] J. C. Ferreira, V. A. Menegatto and C. P. Oliveira, On the nuclearity of integral operators, Positivity 13 (2009), no. 3, 519-541.

[GGK00] I. Gohberg, S. Goldberg And N. Krupnik, Traces and determinants of linear operators, Oper. Theory Adv. Appl. 116, Birkhäuser Verlag, Basel, 2000.

[Gro55] A. Grothendieck, Produits tensoriels topologiques et espaces nucléaires, Mem. Amer. Math. Soc. 1955 (1955), no. 16, 140.

[Hoc66] H. HochSTADT, On the eigenvalues of a class of integral operators arising in laser theory, SIAM Rev. $8(1966), 62-65$.

[HP10] A. HINRICHS AND A. PIETSCH, p-nuclear operators in the sense of Grothendieck, Math. Nachr. 283 (2010), no. 2, 232-261.

[Kön78] H. KÖNIG, Eigenvalues of $p$-nuclear operators, In Proceedings of the International Conference on Operator Algebras, Ideals, and their Applications in Theoretical Physics (Leipzig, 1977), pages 106-113, Teubner, Leipzig, 1978.

[Lid59] V. B. LIDSKIǏ, Non-selfadjoint operators with a trace, Dokl. Akad. Nauk SSSR 125 (1959), $485-487$.

[MO12] V. A. Menegatto AND C. P. Oliveira, Eigenvalue and singular value estimates for integral operators: a unifying approach, Math. Nachr. 285 (2012), no. 17-18, 2222-2232.

[NE05] L. N. TREFETHEN AND M. EmbreE, Spectra and pseudospectra: The behaviour of nonnormal matrices and operators, Princeton University Press, Princeton, NJ, 2005.

[NM64] D. J. NEWMAN AND S. P. MORGan, Existence of eigenvalues of a class of integral equations arising in laser theory, Bell System Tech. J. 43 (1964), 113-126.

[Olo72] R. OLOFF, p-normierte Operatorenideale, Tagungsbericht zur Ersten Tagung der WK Analysis (1970), Beiträge Anal. No. 4, (1972), 105-108.

[Pie80] A. PIETSCH, Operator ideals, Translated from German by the author, North-Holland Mathematical Library 20, North-Holland Publishing Co., Amsterdam-New York, 1980.

[Pie84] A. PIETSCH, Grothendieck's concept of a $p$-nuclear operator, Integral Equations Operator Theory 7 (1984), no. 2, 282-284.

[Pie87] A. Pietsch, Eigenvalues and $s$-numbers, Cambridge Stud. Adv. Math. 13, Cambridge University Press, Cambridge, 1987.

[RL13] O. I. REINOV AND Q. LAIF, Grothendieck-Lidskii theorem for subspaces of $L p$-spaces, Math. Nachr. 286 (2013), no. 2-3, 279-282.

[Sch09] I. SCHUR, Über die charakteristischen Wurzeln einer linearen Substitution mit einer Anwendung auf die Theorie der Integralgleichungen, Math. Ann. 66 (1909), no. 4, 488-510.

[SP61] D. SLEPIAN AND H. O. PollaK, Prolate spheroidal wave functions, Fourier analysis and uncertainty I, Bell System Tech. J. 40 (1961), 43-63.

[Wey49] H. WEYL, Inequalities between the two kinds of eigenvalues of a linear transformation, Proc. Nat. Acad. Sci. U. S. A. 35 (1949), 408-411. 
DEPARTMENT OF MATHEMATICS

IMPERIAL COLLEGE LONDON

180 QUEEN'S GATE

LONDON SW7 2AZ

UNITED KINGDOM

E-mail address: j.delgado@imperial.ac.uk 